\title{
Prognostic value of inflammation-based indexes for intrahepatic cholangiocarcinoma following curative resection
}

\author{
YUNHUA WU ${ }^{1-3}$, FENGGANG REN $^{1-3}$, YICHAO CHAI ${ }^{1-3}$, ZHAO XUE $^{1-3}$, \\ CONG SHEN $^{4}$, XUFENG ZHANG ${ }^{1-3}, \mathrm{YI} \mathrm{LV}^{1-3}$ and LIANGSHUO HU ${ }^{1-3}$ \\ ${ }^{1}$ Department of Hepatobiliary Surgery, First Affiliated Hospital of Xi'an Jiaotong University; \\ ${ }^{2}$ Research Institute of Advanced Surgical Techniques and Engineering, Xi'an Jiaotong University; \\ ${ }^{3}$ Regenerative Medicine and Surgery Engineering Research Center of Shaanxi Province; \\ ${ }^{4}$ Department of Medical Imaging, First Affiliated Hospital of Xi'an Jiaotong University, Xi'an, Shaanxi 710061, P.R. China
}

Received January 28, 2018; Accepted September 6, 2018

DOI: $10.3892 / \mathrm{ol} .2018 .9618$

\begin{abstract}
It is widely acknowledged that inflammatory indices may serve as effective prognosis indicators for various malignancies. In the present study, the prognostic value of systemic inflammatory biomarkers for patients undergoing curative resection for intrahepatic cholangiocellular carcinoma (ICC) was investigated. Clinical data of ICC patients who underwent curative resection between September 2008 and July 2017 were collected. Inflammatory indictors were analyzed using the Area Under the Receiver Operating Characteristic Curve. Indictors that were significantly associated with the overall survival (OS) were used to establish a systemic inflammation-based score system and tested via nomogram using $\mathrm{R}$ software. The neutrophil To lymphocyte ratio (NLR) and lymphocyte to macrophages ratio (LMR) were significantly associated with the OS and disease-free survival of the patients. High NLR and low LMR were associated with worse clinicopathological and survival outcomes. The univariate and multivariate analyses indicated that tumor $\mathrm{T}$ stage, incisal margin, NLR and LMR were associated with the OS of the patients. The systemic inflammation-based scoring system based on LMR and NLR demonstrated a stronger discriminatory capacity and may serve as a useful prognostic parameter for patients undergoing curative resection for ICC. Low LMR and high NLR were observed to be associated with poor prognosis and worse clinical outcomes for patients with ICC undergoing curative surgery. A combined inflammation-based scoring system based on LMR and NLR may effectively predict the outcomes and serve as a novel prognostic predictor for these patients.
\end{abstract}

Correspondence to: Dr Liangshuo Hu, Department of Hepatobiliary Surgery, First Affiliated Hospital of Xi'an Jiaotong University, 277 Yanta West Road, Yanta, Xi'an, Shaanxi 710061, P.R. China E-mail: huliangshuo1983@hotmail.com

Key words: intrahepatic cholangiocellular carcinoma, prognostic factors, inflammation, neutrophil to lymphocyte ratio, lymphocyte to macrophage ratio

\section{Introduction}

Primary liver cancer is one of the most common malignant tumors in the world, with particularly high incidence rates in Asia and Africa $(1,2)$. It is the third leading cause of cancer-associated mortality worldwide (3). The major histopathologic types of liver cancer include hepatocellular carcinoma (HCC) and intrahepatic cholangiocellular carcinoma (ICC) $(2,4)$. Although various methods have been implemented to improve the survival of patients with liver cancer, including surgical resection, liver transplantation, transarterial chemoembolization, local ablation, radiotherapy, chemotherapy and molecular targeted drug treatment, the prognosis remains poor, particularly for ICC (5-8).

ICC is the second most common malignancy worldwide and accounts for $15-20 \%$ of primary liver cancer (9). Due to its rising incidence and poor prognosis, research into ICC is receiving increasing attention $(10,11)$. Surgical resection remains one of the most effective ways to treat $\operatorname{ICC}(12,13)$. However, the clinical outcomes are limited due to the high recurrence and metastasis rates following operation (14). Thus, effective indicators that may predict the prognosis of ICC are essential for the treatment of these patients.

Previous studies have demonstrated that inflammation serves an important role in the development and progression of liver cancer $(15,16)$. It has previously been demonstrated that a number of inflammation-based indicators in the blood are associated with the prognosis of HCC, such as the Glasgow Prognostic Score (GPS), the systemic inflammation score, the neutrophil to lymphocyte ratio (NLR), the lymphocyte to monocyte ratio (LMR), the platelet-to-lymphocyte ratio (PLR), prognostic nutritional index (PNI), prognostic index and red cell distribution width (RDW) (3,17-19). In addition, there are various serum enzyme-associated parameters that have been found to be associated with the clinical outcomes of ICC, including the gamma-glutamyltransferase to platelet ratio (GPR), albumin (ALB) to alkaline phosphatase ratio (APPR), $\gamma$-glutamyltransferase to alanine aminotransferase ratio (GAR) and the ALB to $\gamma$-glutamyltransferase ratio (AGR) $(20,21)$. However, few studies have considered the prognostic significance of serum inflammatory-based indicators for ICC. 
The aims of the present retrospective analysis were to investigate the association between inflammation-based prognostic indicators and the survival of patients undergoing curative surgical resection for ICC.

\section{Materials and methods}

Patients. For this retrospective cohort study, 221 patients who were pathologically diagnosed with ICC in the First Affiliated Hospital of Xi'an Jiaotong University (Xi'an, China) between September 2008 and July 2017 were retrospectively recruited. Patients with active hepatitis, parasitic infection, acute cholangitis or other malignant tumors were excluded. Ultimately, 123 patients following curative resection were enrolled into the study. The institutional ethics committee at the study center approved this study. All participants gave consent after being fully informed of the goal and characteristics of this research.

Treatment and follow-up. Blood tests and computed tomography (CT) scans were routinely performed as preoperative tests within 3 days prior to surgery. The clinical staging was based on The American Joint Committee on Cancer (AJCC) 8th Edition Cancer Staging System (22). Each patient was followed-up at least every 2 months following hospital discharge during the first year and every 3 months thereafter. The final follow-up date was September 30th, 2017.

Demographics and clinical characteristic data. All clinical data were collected from the patients' medical records in the department of Hepatobiliary Surgery of the First Affiliated Hospital of Xi'an Jiaotong University. Clinical data included age, gender, tumor size, number of nodules and presence or absence of vascular invasion. Furthermore, preoperative biochemical indices were measured, including white blood cell counts (WBC), platelet counts (PLT), neutrophil counts, lymphocyte counts, megakaryocyte counts, RDW, $\alpha$-fetoprotein (AFP) levels, alanine transaminase (ALT) levels, aspartate aminotransferase (AST) levels, total bilirubin (TBIL) levels, indirect bilirubin (IBIL) levels, alkaline phosphatase (ALP) levels, ALB levels, hepatitis B surface antigen (HbsAg) levels and hepatitis B virus deoxyribonucleic acid (HBV-DNA) load. NLR was defined as the neutrophil count/lymphocyte count ratio; dNLR was calculated by neutrophil count/(WBC-neutrophil counts) ratio; LMR was defined as the lymphocyte count/megakaryocyte count ratio; PLR was defined as the platelet count/lymphocyte count ratio; PNI was defined as ALB + (5x lymphocyte count). The primary endpoints of this study were overall survival time (OS) and disease-free survival time (DFS). OS was defined as the time between radical surgery and mortality. DFS was defined as the time between radical surgery and tumor recurrence.

Statistical methods. Statistical analyses were performed using SPSS version 18.0 (SPSS Inc., Chicago, IL, USA). Continuous data are presented as the mean \pm standard deviation and were compared using a unpaired Student's t-test or one-way analysis of variance for normal distribution data with Fisher's LSD post hoc test for the comparison of among different groups, Kruskal-Wallis test was used for multi-group comparison of abnormal distribution. The categorical variables were compared using a $\chi^{2}$ test and a Fisher's exact test. The diagnostic accuracy of all the indicators was determined using receiver operating characteristic (ROC) curve analysis. Indicators that displayed significance ere chosen for the next part of the study and the Youden's index was applied to determine the optimal cut-off values (22). Patients were divided into different groups according to these aforementioned cut-off values. Univariate analysis of variables associated with survival was performed using log-rank testing to evaluate clinical factors associated with OS. Multivariate analysis was performed using Cox proportional hazards regression modelling using backward elimination and likelihood ratio testing, and the included variables were those which had significant associations with OS, determined by the univariate analysis. The inflammation-based scoring system was defined as follows: Patients with high NLR and high LMR were assigned a score of 2; patients with high NLR and low LMR or low NLR and high LMR were assigned as score of 1; patients who had low NLR and LMR were assigned a score of 0 . Patients were grouped according to this score. The Kaplan-Meier method was used to analyze the long-term effect of the different groups and these were compared using the log-rank test. Finally, nomograms were used to validate the outcomes. Nomograms for possible prognostic factors associated with OS were using $\mathrm{R}$ software 3.4.0 (Institute for Statistics and Mathematics, Vienna, Austria). and the model performance for predicting outcome was evaluated by Harrell's concordance index (c-index), as previously described (23). $\mathrm{P}<0.05$ was considered to indicate a statistically significant difference.

\section{Results}

Patients' characteristics. A total of 123 patients were recruited to the present study, who had been pathologically diagnosed with ICC and undergone radical resection between September 2008 and July 2017, including 67 males and 56 females. The mean age of the patients was $56.80 \pm 10.67$ (29-79) years old. The final follow-up date was September 30th, 2017. The median follow-up time was 29.1 months (2-64 months). Following analysis with the Area Under the ROC curve (AUROC), NLR and LMR only were found to be significantly associated with the OS of patients (Fig. 1, data for indictors which were not associated with OS not presented). The optimal cut-off value of OS for LMR and NLR was 3.42 and 2.05 , respectively.

According to the cut-off value of NLR and LMR, the cohort was divided into lower and higher groups as presented in Table I. Higher NLR was observed to be associated with a higher ratio of male patients, larger tumor diameter, higher lymph node metastasis rate and increased white blood cell and megakaryocyte counts. Lower LMR was associated with larger tumor diameter, higher lymph node metastasis rate, increased numbers of patients at tumor stage 3-4 (T3-4) and Tumor, Node, Metastasis (TNM) stage 3-4, lower ALB levels and increased megakaryocyte counts.

Survival outcomes. The 1-year and 3-year OS for the whole cohort were 37.40 and $5.69 \%$, respectively. The median OS was 9 months. Higher NLR was associated with poor OS and decreased DFS. Patients with higher LMR exhibited higher 3-year OS and 3-year DFS (Fig. 2). 
Table I. Associations between clinical characteristics of NLR and LMR.

\begin{tabular}{|c|c|c|c|c|c|c|}
\hline Parameter & $\begin{array}{c}\mathrm{NLR} \leq 2.05 \\
(\mathrm{n}=25)\end{array}$ & $\begin{array}{l}\text { NLR }>2.05 \\
\quad(n=98)\end{array}$ & P-value & $\begin{array}{l}\mathrm{LMR} \leq 3.42 \\
\quad(\mathrm{n}=57)\end{array}$ & $\begin{array}{l}\mathrm{LMR}>3.42 \\
\quad(\mathrm{n}=66)\end{array}$ & P-value \\
\hline Age, year ${ }^{b}$ & $56.08 \pm 11.81$ & $56.99 \pm 10.42$ & 0.705 & $56.58 \pm 11.06$ & $57.00 \pm 10.41$ & 0.828 \\
\hline Sex (male/female) & $8 / 17$ & $59 / 39$ & $0.011^{\mathrm{a}}$ & $32 / 25$ & $35 / 31$ & 0.730 \\
\hline BMI, $\mathrm{kg} / \mathrm{m}^{2 \mathrm{~b}}$ & $23.00 \pm 4.24$ & $23.13 \pm 3.70$ & 0.878 & $22.50 \pm 3.91$ & $23.63 \pm 3.64$ & 0.101 \\
\hline Tumor diameter, $\mathrm{cm}^{\mathrm{b}}$ & $4.81 \pm 2.63$ & $6.46 \pm 3.18$ & $0.018^{\mathrm{a}}$ & $6.86 \pm 3.18$ & $5.48 \pm 2.98$ & $0.015^{\mathrm{a}}$ \\
\hline Differentiated (well/poorly) & $17 / 8$ & $53 / 45$ & 0.152 & $28 / 29$ & $42 / 24$ & 0.105 \\
\hline $\begin{array}{l}\text { Incisal margin } \\
\text { (negative/positive) }\end{array}$ & $13 / 12$ & $63 / 35$ & 0.259 & $34 / 23$ & $42 / 24$ & 0.650 \\
\hline $\mathrm{N}(-/+)$ & $22 / 3$ & $53 / 45$ & $0.002^{\mathrm{a}}$ & $25 / 32$ & $50 / 16$ & $0.000^{\mathrm{a}}$ \\
\hline $\mathrm{T}(1-2 / 3-4)$ & $17 / 8$ & $51 / 47$ & 0.152 & $25 / 32$ & $43 / 23$ & $0.018^{\mathrm{a}}$ \\
\hline TNM stage (I-II/III-IV) & $11 / 14$ & $27 / 71$ & 0.112 & $10 / 47$ & $28 / 38$ & $0.003^{\mathrm{a}}$ \\
\hline $\begin{array}{l}\text { Vascular invasion } \\
\text { (absent/present) }\end{array}$ & $18 / 7$ & $63 / 35$ & 0.468 & $34 / 23$ & $47 / 19$ & 0.177 \\
\hline WBC count, $\times 10^{3} / \mathrm{ml}^{\mathrm{b}}$ & $5.75 \pm 2.23$ & $7.19 \pm 2.71$ & $0.015^{\mathrm{a}}$ & $7.37 \pm 2.86$ & $6.49 \pm 2.47$ & 0.071 \\
\hline Platelet count, $\times 10^{3} / \mathrm{ml}^{\mathrm{b}}$ & $178.96 \pm 83.70$ & $198.01 \pm 93.65$ & 0.356 & $201.40 \pm 82.81$ & $187.86 \pm 98.96$ & 0.416 \\
\hline Albumin, $\mathrm{g} / \mathrm{dl}^{\mathrm{b}}$ & $39.05 \pm 5.14$ & $38.07 \pm 5.83$ & 0.455 & $36.58 \pm 5.21$ & $39.73 \pm 5.72$ & $0.002^{\mathrm{a}}$ \\
\hline $\begin{array}{l}\text { Neutrophil count, } \\
\mathrm{x} 10^{3} / \mathrm{ml}^{\mathrm{b}}\end{array}$ & $6.51 \pm 14.33$ & $4.82 \pm 2.18$ & 0.562 & $5.13 \pm 2.46$ & $5.20 \pm 8.86$ & 0.952 \\
\hline $\begin{array}{l}\text { Lymphocyte count, } \\
\mathrm{x} 10^{3} / \mathrm{ml}^{\mathrm{b}}\end{array}$ & $2.38 \pm 5.23$ & $1.47 \pm 0.52$ & 0.391 & $1.36 \pm 0.45$ & $1.91 \pm 3.23$ & 0.203 \\
\hline $\begin{array}{l}\text { Megakaryocyte count, } \\
\times 10^{3} / \mathrm{ml}^{\mathrm{b}}\end{array}$ & $0.33 \pm 0.17$ & $0.48 \pm 0.21$ & $0.001^{\mathrm{a}}$ & $0.54 \pm 0.22$ & $0.37 \pm 0.17$ & $0.000^{\mathrm{a}}$ \\
\hline $\mathrm{TBIL}, \mathrm{mmol} / \mathrm{l}^{\mathrm{b}}$ & $32.92 \pm 57.42$ & $38.12 \pm 68.62$ & 0.728 & $37.57 \pm 65.67$ & $36.62 \pm 67.36$ & 0.937 \\
\hline $\mathrm{AST}, \mathrm{U} / \mathrm{l}^{\mathrm{b}}$ & $66.62 \pm 61.00$ & $133.21 \pm 659.63$ & 0.616 & $163.25 \pm 798.85$ & $69.23 \pm 106.15$ & 0.380 \\
\hline $\mathrm{ALT}, \mathrm{U} / \mathrm{l}^{\mathrm{b}}$ & $68.20 \pm 68.92$ & $131.17 \pm 545.37$ & 0.567 & $152.14 \pm 652.15$ & $79.28 \pm 148.61$ & 0.411 \\
\hline $\mathrm{AFP}, \mathrm{ng} / \mathrm{ml}^{\mathrm{b}}$ & $12.34 \pm 40.56$ & $31.37 \pm 157.13$ & 0.551 & $3.16(1.21-1,440)$ & $3.11(1.22-420)$ & 0.563 \\
\hline CA-199 kU/1 (median) & $34.04(0.6-10,000)$ & $185(7.74-10,000)$ & 0.065 & $185(3.55-10,000)$ & $61(0.6-10,000)$ & 0.001 \\
\hline GGT, $\mathrm{U} / \mathrm{l}^{\mathrm{b}}$ & $162.61 \pm 187.45$ & $241.74 \pm 347.26$ & 0.275 & $251.52 \pm 396.68$ & $195.71 \pm 204.48$ & 0.320 \\
\hline Child-plug score $(\mathrm{A}, \mathrm{B} / \mathrm{C})$ & $19 / 6$ & $68 / 30$ & 0.517 & $37 / 20$ & $50 / 16$ & 0.187 \\
\hline
\end{tabular}

${ }^{\mathrm{a}} \mathrm{P}<0.05,{ }^{\mathrm{b}}$ mean \pm standard deviation. NLR, neutrophil to lymphocyte ratio; LMR, lymphocyte to macrophage ratio; BMI, body mass index; TNM, Tumor, Node, Metastasis; WBC, white blood cell; TBIL, total bilirubin levels; AST, aspartate aminotransferase; ALT, alanine aminotransferase; AFP, $\alpha$-fetoprotein; CA-199, cancer antigen 199; GGT, $\gamma$-glutamyltransferase.
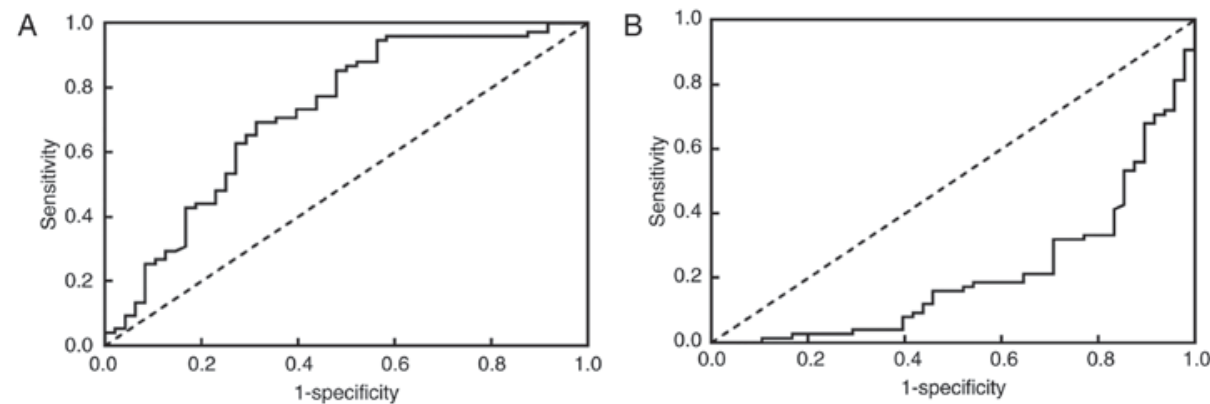

Figure 1. ROC analysis curve for NLR and LMR. (A) The ROC curve of NLR, $\mathrm{P}<0.05$ and AUC=0.645. The cut-off point is 2.05 . (B) The ROC curve of LMR, $\mathrm{P}<0.05$ and $\mathrm{AUC}=0.299$. The cut-off point is 3.41. ROC, receiver operating characteristic; NLR, neutrophil to lymphocyte ratio; LMR, lymphocyte to macrophage ratio; AUC, area under the curve.

The univariate analysis of OS-associated indicators are presented in Table II. Tumor diameter, degree of tumor differentiation, lymph node metastasis, resection margin,
T stages, TNM stage, vascular invasion, NLR, LMR and macrophage counts were found to be associated with the OS for the cohort. In order to exclude the colinear regression 
Table II. Univariate and Multivariate analysis of the clinical characteristic factors associated with OS.

\begin{tabular}{|c|c|c|c|c|c|c|}
\hline \multirow[b]{2}{*}{ Parameter } & \multicolumn{3}{|c|}{ Univariate analysis } & \multicolumn{3}{|c|}{ Multivariate analysis } \\
\hline & P-value & HR & $95 \% \mathrm{CI}$ & P-value & HR & $95 \% \mathrm{CI}$ \\
\hline \multicolumn{7}{|l|}{ Age (years) } \\
\hline$<60 ; \geq 60$ & 0.588 & 1.145 & $0.701-1.872$ & - & - & - \\
\hline \multicolumn{7}{|l|}{ Sex } \\
\hline Male; female & 0.459 & 0.829 & $0.504-1.362$ & - & - & - \\
\hline \multicolumn{7}{|l|}{ BMI $\left(\mathrm{kg} / \mathrm{m}^{2}\right)$} \\
\hline$<24 ; \geq 24$ & 0.615 & 0.879 & $0.532-1.452$ & - & - & - \\
\hline \multicolumn{7}{|l|}{ Tumor diameter } \\
\hline \multicolumn{7}{|l|}{ Differentiated } \\
\hline \multicolumn{7}{|l|}{ Incisal margin } \\
\hline \multicolumn{7}{|l|}{$\mathrm{N}$} \\
\hline $\mathrm{N}-; \mathrm{N}+$ & $0.007^{\mathrm{a}}$ & 1.990 & $1.204-3.288$ & 0.091 & 1.642 & $0.924-2.918$ \\
\hline \multicolumn{7}{|l|}{$\mathrm{T}$} \\
\hline T1-2; T3-4 & $0.006^{\mathrm{a}}$ & 2.027 & $1.228-3.347$ & $0.014^{\mathrm{a}}$ & 2.015 & $1.155-3.516$ \\
\hline \multicolumn{7}{|l|}{ TNM stage } \\
\hline \multicolumn{7}{|l|}{ Vascular invasion } \\
\hline \multicolumn{7}{|l|}{ NLR } \\
\hline \multicolumn{7}{|l|}{ LMR } \\
\hline$\leq 3.42 ;>3.42$ & $0.000^{\mathrm{a}}$ & 0.686 & $0.547-0.819$ & $0.023^{\mathrm{a}}$ & 0.789 & $0.643-0.968$ \\
\hline WBC count $\left(\mathrm{x} 10^{3} / \mathrm{ml}\right)$ & 0.056 & 1.093 & 0.998-1.197 & - & - & - \\
\hline \multicolumn{7}{|l|}{ Platelet count $\left(\times 10^{3} / \mathrm{ml}\right)$} \\
\hline$<100 ; \geq 100$ & 0.253 & 1.494 & $0.751-2.973$ & - & - & - \\
\hline \multicolumn{7}{|l|}{ Albumin (g/dl) } \\
\hline$<35 ; \geq 35$ & 0.363 & 0.778 & $0.453-1.337$ & - & - & - \\
\hline Neutrophil count $\left(\mathrm{x} 10^{3} / \mathrm{ml}\right)$ & 0.685 & 1.011 & $0.964-1.060$ & - & - & - \\
\hline Lymphocyte count $\left(\mathrm{x} 10^{3} / \mathrm{ml}\right)$ & 0.769 & 1.020 & $0.896-1.161$ & - & - & - \\
\hline Macrophages count $\left(\times 10^{3} / \mathrm{ml}\right)$ & $0.009^{\mathrm{a}}$ & 4.173 & $1.435-12.133$ & 0.279 & 2.064 & $0.556-7.665$ \\
\hline \multicolumn{7}{|l|}{ TBIL (mmol/l) } \\
\hline$<20.5 ; \geq 20.5$ & 0.441 & 1.223 & $0.733-2.038$ & - & - & - \\
\hline \multicolumn{7}{|l|}{$\operatorname{AST}(\mathrm{U} / \mathrm{l})$} \\
\hline$<45 ; \geq 45$ & 0.580 & 0.866 & $0.520-1.433$ & - & - & - \\
\hline \multicolumn{7}{|l|}{$\operatorname{ALT}(\mathrm{U} / \mathrm{l})$} \\
\hline$<45 ; \geq 45$ & 0.296 & 0.756 & $0.447-1.278$ & - & - & - \\
\hline \multicolumn{7}{|l|}{$\operatorname{AFP}(n g / m l)$} \\
\hline$<400 ; \geq 400$ & 0.403 & 1.297 & $0.705-2.386$ & - & - & - \\
\hline \multicolumn{7}{|l|}{ CA-199 (kU/l) } \\
\hline$<35 ; \geq 35$ & 0.789 & 0.928 & $0.537-1.603$ & - & - & - \\
\hline \multicolumn{7}{|l|}{ Child-plug score } \\
\hline $\mathrm{A} ; \mathrm{B} ; \mathrm{C}$ & 0.422 & 1.207 & $0.763-1.911$ & - & - & - \\
\hline \multicolumn{7}{|l|}{ ALP (U/1) } \\
\hline$<100 ; \geq 100$ & 0.249 & 1.357 & $0.808-2.278$ & - & - & \\
\hline GGT (U/1) & & & & & & \\
\hline$<50 ; \geq 50$ & 0.277 & 1.370 & $0.777-2.414$ & - & - & - \\
\hline
\end{tabular}

${ }^{\mathrm{a}} \mathrm{P}<0.05$. ${ }^{\mathrm{b}} \mathrm{TNM}$ stages were excluded to avoid the influence of colinear regression. OS, overall survival; NLR, neutrophil to lymphocyte ratio; LMR, lymphocyte to macrophage ratio; BMI, body mass index; TNM, Tumor, Node, Metastasis; WBC, white blood cell; TBIL, total bilirubin levels; AST, aspartate aminotransferase; ALT, alanine aminotransferase; AFP, $\alpha$-fetoprotein; CA-199, cancer antigen 199; GGT, $\gamma$-glutamyltransferase; CI, confidence interval; HR, hazard ratio. 

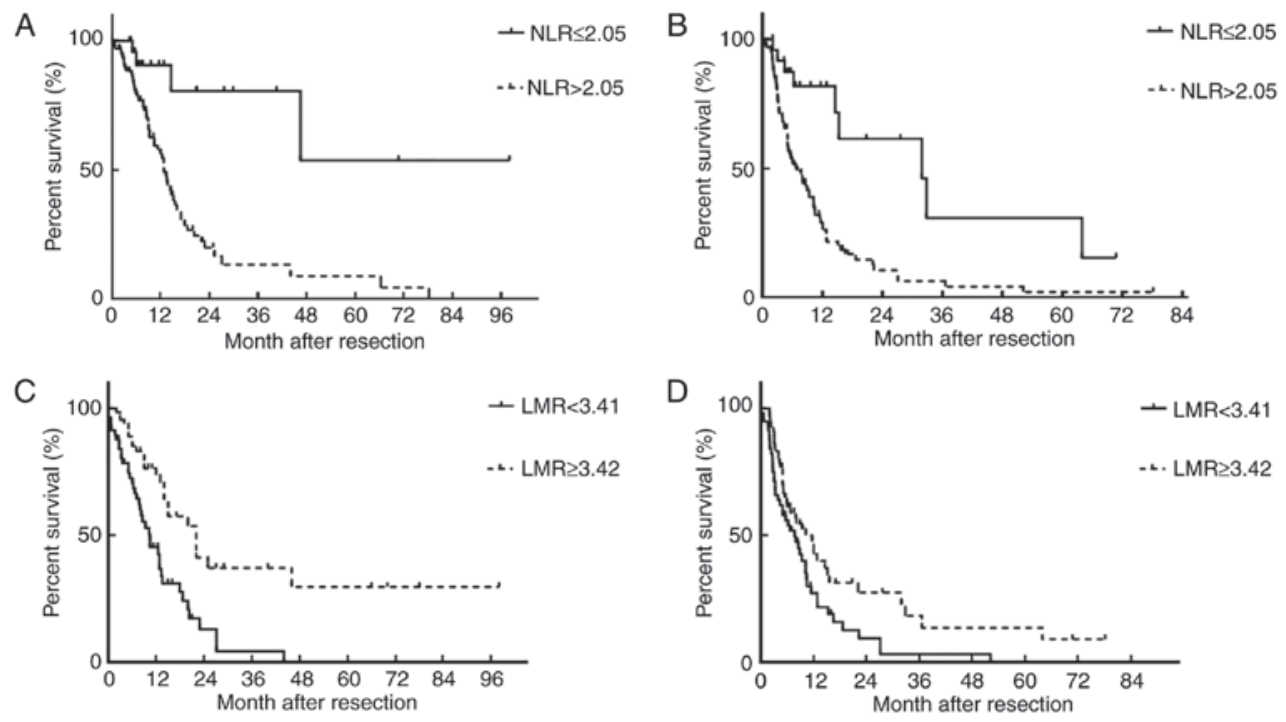

Figure 2. OS and DFS of patients with different NLR and LMR scores. (A) The OS of patients with low NLR compared with high NLR; P<0.001. (B) The DFS of patients with low NLR compared with high NLR; P $<0.001$. (C) The OS of patients with low LMR compared with high LMR; P $<0.001$. (D) The DFS of patients with low LMR compared with high LMR; P<0.001. OS, overall survival rate; DFS, disease-free survival rate; NLR, neutrophil to lymphocyte ratio; LMR, lymphocyte to macrophage ratio.
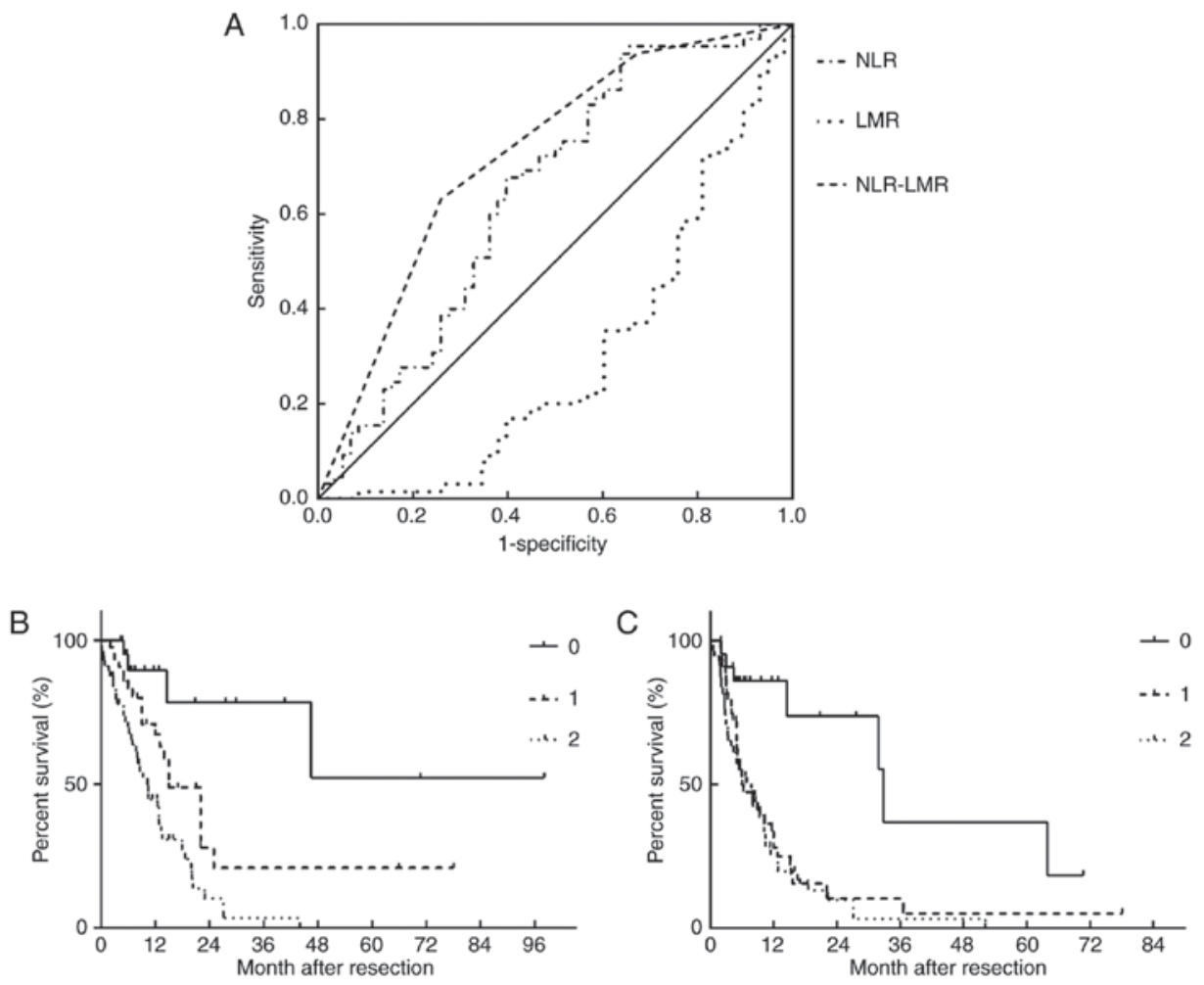

Figure 3. Comparison of the AUROC values and long-term effect on the OS of ICC patients based on inflammatory scores system. (A) The ROC curve for NLR, LMR and inflammatory-based scoring system; AUROC values were $0.645,0.299$ and 0.724 , respectively. (B) A comparison of the OS between the different groups based on the inflammatory-based system; $\mathrm{P}<0.001$. (C) A comparison of the DFS between the different groups based on the inflammatory-based system; $\mathrm{P}<0.001$. AUROC, Area Under the Receiver Operating Characteristic Curve; OS, overall survival rate; ICC, intrahepatic cholangiocellular carcinoma; NLR, neutrophil to lymphocyte ratio; LMR, lymphocyte to macrophage ratio.

amongst the related factors, TNM stages were not included in the multivariate analysis. The results showed that the T stage, resection margin, NLR and LMR were significantly associated with the OS of patients with ICC.

To comprehensively evaluate the association between the inflammation-based score and the OS, patients were divided into three groups: Score 0 group ( $n=23)$; score 1 group $(n=44)$; and score 2 group $(n=56)$. The ROC analysis was used to determine the discriminatory capacity of the NLR, LMR and inflammation-based score system, as presented in Fig. 3A. The AUROC of NLR, LMR and the inflammation-based scoring system was $0.645,0.299$ and 0.724 , respectively. 
Table III. Association between clinical characteristics and the inflammation-based scoring system.

\begin{tabular}{|c|c|c|c|c|}
\hline \multirow[b]{2}{*}{ Parameter } & \multicolumn{3}{|c|}{ Inflammation score } & \multirow[b]{2}{*}{ P-value } \\
\hline & $\begin{array}{c}0 \\
\mathrm{~N}=23\end{array}$ & $\begin{array}{c}1 \\
\mathrm{~N}=44\end{array}$ & $\begin{array}{c}2 \\
\mathrm{~N}=56\end{array}$ & \\
\hline Ages, years ${ }^{\mathrm{f}}$ & $55.65 \pm 12.21$ & $58.05 \pm 9.25$ & $56.30 \pm 11.15$ & 0.615 \\
\hline Sex (male/female) & $8 / 15$ & 26/18 & $33 / 23$ & 0.110 \\
\hline $\mathrm{BMI}, \mathrm{kg} / \mathrm{m}^{2 \mathrm{f}}$ & $23.12 \pm 4.41$ & $23.87 \pm 3.16$ & $22.51 \pm 3.94$ & 0.208 \\
\hline Tumor diameter, $\mathrm{cm}^{\mathrm{f}}$ & $4.83 \pm 2.70^{\mathrm{d}}$ & $5.83 \pm 3.05$ & $6.88 \pm 3.21^{\mathrm{d}}$ & $0.023^{\mathrm{a}}$ \\
\hline Differentiated (well/poorly) & $15 / 8$ & $27 / 17$ & $28 / 28$ & 0.351 \\
\hline Incisal margin (negative/positive) & $20 / 3$ & $29 / 15$ & $27 / 29$ & $0.004^{\mathrm{a}}$ \\
\hline $\mathrm{N}(-/+)$ & $19 / 4$ & $31 / 13$ & $25 / 31$ & $0.002^{\mathrm{a}}$ \\
\hline $\mathrm{T}(1-2 / 3-4)$ & $15 / 8$ & $29 / 15$ & $24 / 32$ & $0.040^{\mathrm{a}}$ \\
\hline TNM stage (I-II/III-IV) & $11 / 12$ & $19 / 25$ & $8 / 48$ & $0.001^{\mathrm{a}}$ \\
\hline Vascular invasion (absent/present) & $17 / 6$ & $30 / 14$ & $34 / 22$ & 0.490 \\
\hline WBC count, $\times 10^{3} / \mathrm{ml}^{\mathrm{f}}$ & $5.57 \pm 2.22^{\mathrm{c}, \mathrm{d}}$ & $6.99 \pm 2.44^{\mathrm{c}}$ & $7.38 \pm 2.89^{\mathrm{d}}$ & $0.022^{\mathrm{a}}$ \\
\hline Platelet count, $\times 10^{3} / \mathrm{ml}^{\mathrm{f}}$ & $178.17 \pm 87.22$ & $191.00 \pm 103.41$ & $203.16 \pm 83.99$ & 0.528 \\
\hline Albumin, $\mathrm{g} / \mathrm{dl}^{\mathrm{f}}$ & $39.87 \pm 4.02$ & $39.06 \pm 6.83$ & $36.99 \pm 5.07$ & 0.064 \\
\hline Neutrophil count, $\mathrm{x} 10^{3} / \mathrm{ml}^{\mathrm{f}}$ & $2.45 \pm 5.46^{\mathrm{d}}$ & $4.40 \pm 1.57$ & $5.17 \pm 2.49^{\mathrm{d}}$ & $0.007^{\mathrm{a}}$ \\
\hline Lymphocyte count, $\mathrm{x} 10^{3} / \mathrm{ml}^{\mathrm{f}}$ & $2.85 \pm 6.35^{\mathrm{c}, \mathrm{d}}$ & $1.64 \pm 0.55^{\mathrm{c}}$ & $1.34 \pm 0.44^{\mathrm{d}}$ & $0.012^{\mathrm{a}}$ \\
\hline Megakaryocyte, $\times 10^{3} / \mathrm{ml}^{\mathrm{f}}$ & $0.31 \pm 0.17^{\mathrm{d}}$ & $0.40 \pm 0.16^{\mathrm{e}}$ & $0.54 \pm 0.22^{\mathrm{d}, \mathrm{e}}$ & $0.000^{\mathrm{a}}$ \\
\hline $\mathrm{TBIL}, \mathrm{mmol} / \mathrm{l}^{\mathrm{f}}$ & $25.57 \pm 43.09$ & $45.99 \pm 85.16$ & $34.77 \pm 61.13$ & 0.463 \\
\hline $\mathrm{AST}, \mathrm{U} / \mathrm{l}^{\mathrm{f}}$ & $59.97 \pm 57.28$ & $80.69 \pm 123.95$ & $67.69 \pm 106.11$ & 0.716 \\
\hline ALT, $U / 1^{\mathrm{f}}$ & $64.86 \pm 69.91$ & $196.06 \pm 796.56$ & $79.31 \pm 149.86$ & 0.420 \\
\hline $\mathrm{AFP}, \mathrm{ng} / \mathrm{ml}^{\mathrm{f}}$ & $3.06(1.41-206.00)$ & $3.12(1.22-420.66)$ & $3.16(1.21-1,440)$ & $0.555^{\mathrm{b}}$ \\
\hline CA-199, kU/l (median) & $44.08(0.6-10,000)$ & $95.11(0.8-10,000)$ & $185(3.55-10,000)$ & $0.697^{b}$ \\
\hline $\mathrm{GGT}, \mathrm{U} / \mathrm{l}^{\mathrm{f}}$ & $154.49 \pm 182.39$ & $290.97 \pm 458.74$ & $203.54 \pm 215.42$ & 0.204 \\
\hline Child-plug score (A, B/C) & $20 / 3$ & $30 / 14$ & $37 / 19$ & 0.161 \\
\hline
\end{tabular}

${ }^{\mathrm{a} P}<0.05$. ${ }^{\mathrm{b}} \mathrm{Kruskal}-$ Wallis test. BMI, body mass index; TNM, Tumor, Node, Metastasis; WBC, white blood cell; TBIL, total bilirubin levels; AST, aspartate aminotransferase; ALT, alanine aminotransferase; AFP, $\alpha$-fetoprotein; CA-199, cancer antigen 199; GGT, $\gamma$-glutamyltransferase. ${ }^{c}$ Represent group Inflammation score 0 has significance with group Inflammation score 1 ; ' represent group Inflammation score 0 has significance with group Inflammation score 3; ${ }^{\mathrm{e}}$ represent group Inflammation score 1 has significance with group Inflammation score $2 .{ }^{\mathrm{f}} \mathrm{Mean} \pm$ standard deviation.

When comparing the OS and DFS rates of different groups based on the inflammation-based score system, patients in the higher score group had worse prognosis, as presented in Fig. 3B and C.

The clinical characteristics of different inflammatory-based score groups were further compared. The three groups exhibited differences in the tumor diameter, incisal margin, lymph node metastasis, T stage, TNM stage, serum ALB level, white blood cell count, and lymphocyte and megakaryocyte counts (Table III).

To verify the results, a nomogram was established, using the indicators that were significantly associated with the OS. The results were identified to be the same as those for the Cox regression analysis (Fig. 4A). The Harrell's c-indexes of the nomograms for prediction of the OS of patients with ICC were 0.74 (95\% CI: 0.677-0.803). Calibration curves for 1-year, 2-year and 3-year nomograms (Fig 4B-D) revealed no deviations from the reference line and no need for recalibration. Thus, the nomogram verified that the NLR and LMR may serve as effective indicators for the prognosis of ICC.

\section{Discussion}

Inflammation has long been reported to be associated with the development and progression of liver cancer (24). Inflammation may contribute to the cancer microenvironment and promote the proliferation of cancer cells $(25,26)$. The cell-mediated componenet of the immune system serves an important role in the immune response to a tumor. Levels of peripheral blood cells, such as WBCs, neutrophils granulocytes and lymphocytes, may reflect the inflammatory status of patients (27). It has previously been reported that high numbers of tumor-infiltrating lymphocytes correlate with better prognosis in patients with breast cancer (28). Neutrophils are capable of producing cytokines and chemokines, including vascular endothelial growth factor (VEGF), which may promote tumor angiogenesis and cancer cell proliferation, whilst acting to suppress lymphocyte-mediated cytolysis $(29,30)$. Furthermore, megakaryocyte and platelet numbers have been reported to be associated with a cancer-promoting environment. Increasing evidence has demonstrated that serum inflammatory indicators, 
A

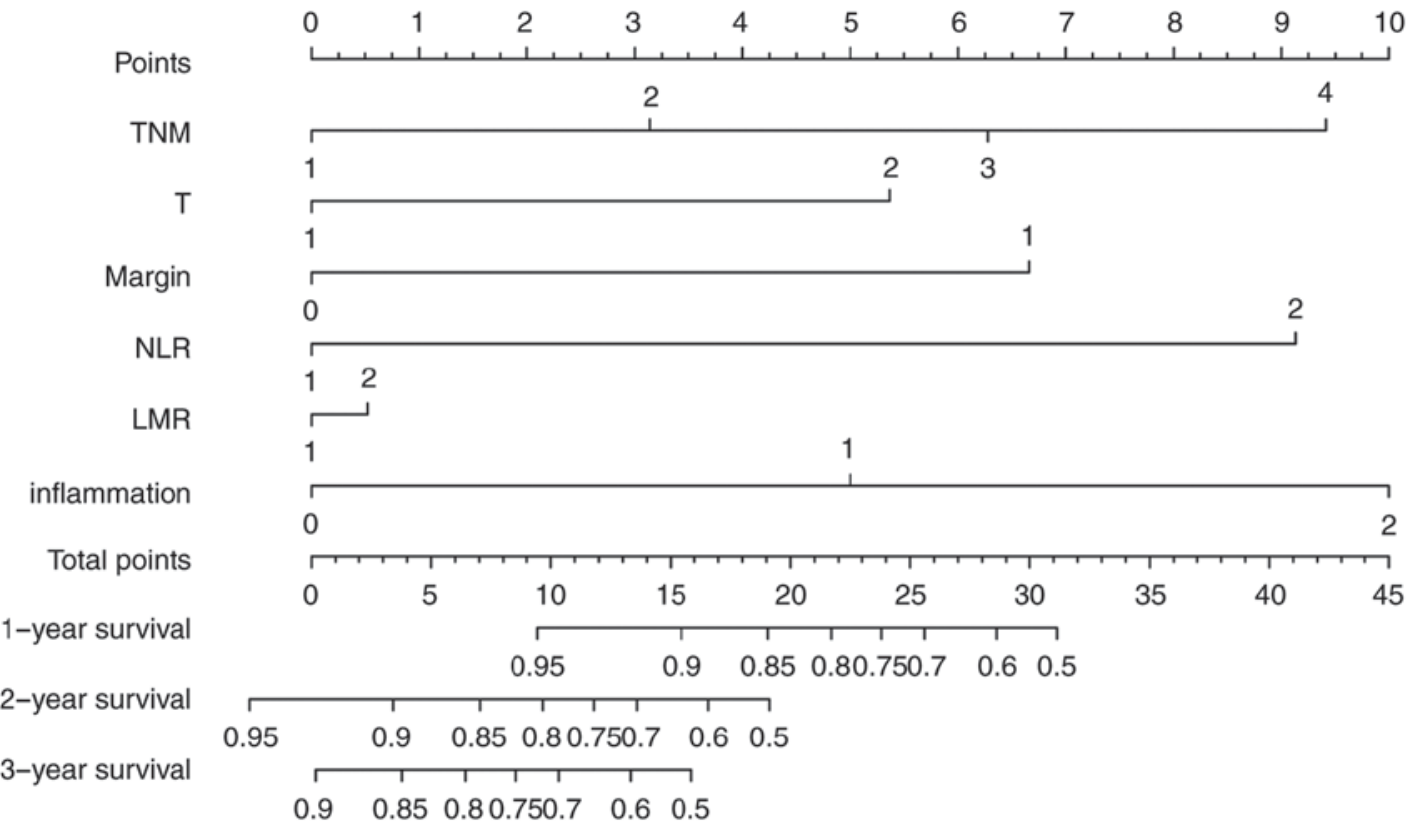

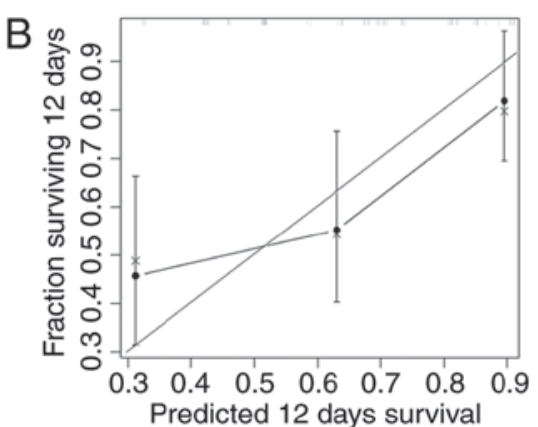

$n=123 d=65 p=6,41 \quad X$-resampling oplimism subjects per group added, $\mathrm{B}=74$ Gray:ideal

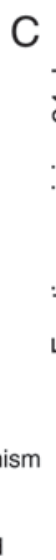

$$
\text { 政 }
$$

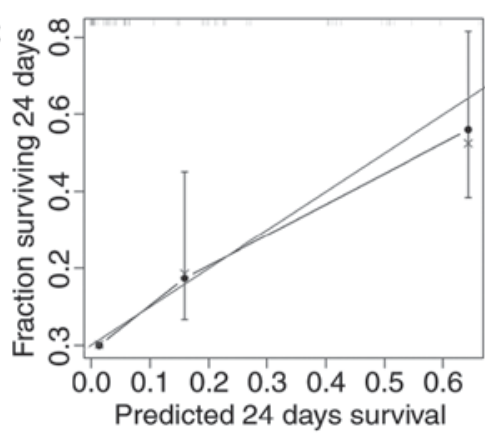

$\begin{array}{ll}\mathrm{n}=123 \mathrm{~d}=65 \mathrm{p}=6,41 & \mathrm{X} \text {-resampling oplimism } \\ \text { subjects per group } & \text { added, } 82\end{array}$ $\begin{array}{ll}\text { subjects per group } & \text { added, 82 } \\ \text { Gray:ideal } & \text { Based on }\end{array}$ observed-predicted

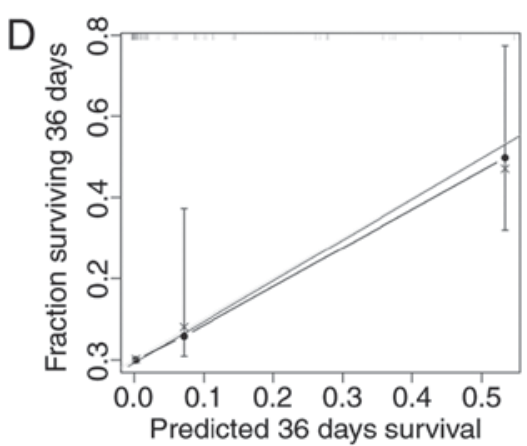

$\begin{array}{ll}\mathrm{n}=123 \mathrm{~d}=65 \mathrm{p}=6,41 & \text { X-resampling } \\ \text { subjects per group } & \text { oplimism added, } \\ \text { Gray:ideal } & \mathrm{B}=80 \\ & \text { Based on } \\ & \text { observed-predicted }\end{array}$

Figure 4. Nomograms of the parameters for the OS of patients undergoing curative resection for ICC. (A) Nomograms established a prognostic model using clinicopathological characteristics and pretreatment inflammatory biomarkers to predict OS for ICC. Nomograms may be interpreted by summing up the points assigned to each variable, which is indicated at the top of scale. The total points may be converted to predict the 3 -year probability of mortality and recurrence or metastasis for a patient in the lowest scale. The Harrell's c-indexes for OS prediction were 0.74 (95\% CI: $0.677-0.803$ ). Calibration curves for (B) 1-year OS, (C) 2-year OS and (D) 3-year OS using nomograms with clinicopathological characteristics and pretreatment inflammatory biomarkers are presented. The $\mathrm{x}$-axis is the nomogram-predicted probability of survival and $\mathrm{y}$-axis is actual survival. The reference line is 98 and indicates perfect calibration. OS, overall survival rate; ICC, intrahepatic cholangiocellular carcinoma; NLR, neutrophil to lymphocyte ratio; LMR, lymphocyte to macrophage ratio; TNM, Tumor, Node, Metastasis.

such as NLR, PLR, LMR, RDW and PNI, are associated with the prognosis of various cancer types $(3,19,31,32)$.

Although the association between inflammatory-based indicators and HCC has been extensively studied, little is known about the usefulness of these indicators in ICC. In the present study, it was revealed that NLR, LMR and the inflammation-based score based on these may serve as useful indicators in the prognosis of patients with ICC. Patients with lower LMR, higher NLR or higher inflammation scores may have worse pathological and clinical outcomes.

NLR as a prognostic factor for liver cancer has been widely reported (33-35). It was demonstrated to be associated with worse clinicopathological characteristics and it is also been reported to be an independent predictor of long-term survival for various malignant tumors $(32,33,35,36)$. In the present study, the optimal cut-off value of NLR was 2.05. In the multivariate analysis, the hazard ratio was 1.033 (95\% CI, 1.001-1.067; $\mathrm{P}<0.05)$, which is concordant with previous studies $(17,37)$. Patients with higher NLR exhibited tumors of larger diameters and at more advanced stages, which is consistent with previous studies $(3,38,39)$.

LMR is a favorable prognostic factor for clinical outcomes in patients with HCC (24). In the present study it has been identified that LMR is also associated with the prognosis of ICC. Lymphocytes and monocytes are vital for the development and prognosis of various cancer types and are involved in the development of tumors through the release of various soluble factors, which may be essential for tumor angiogenesis, invasion and metastasis $(40,41)$. In the present study, lower LMR was demonstrated to be associated with worse prognostic and 
clinical outcomes. Although studies have previously reported LMR to be an independent factor for HCC, this is, to the best of our knowledge, the first evidence to suggest that lower LMR correlates with worse prognosis, therefore may be a potential clinical indicator for patients with ICC $(24,42)$.

The current study identified NLR and LMR to be better predictors compared with other inflammatory indicators for patients with ICC. By combining the two indicators together, it was discovered that the prognostic significance of the inflammatory-based system was improved compared with the simple use of a single index. With AUROC analysis, this inflammatory model had a stronger predictive ability compared with NLR or LMR alone. On the basis of this inflammatory score model, it was also discovered that the higher scoring groups had worse prognostic and clinicopathological outcomes.

Many studies have reported $\gamma$-glutamyl transferase (GGT)-associated enzymes, including AGR, GPR and GAR, along with other indicators such as PNI, to be associated with the prognosis of ICC $(20,43,44)$. However, the present study demonstrated that these parameters were not significantly associated with the prognosis of ICC. This may be due to the fact that the tumors of the enrolled patients were at a later stage compared with previous studies. The patients in this cohort displaced high serum GGT levels and low serum PNI levels. High GGT levels may reflect disorders of the bile tract whilst low PNI may reflect the nutrition state of the patient, however these indicators may not be suitable as OS predictors for patients in the later tumor stage.

Currently, a lot of studies have established various nomograms using the risk factors for the survival and recurrence of the ICC patients (9). In 2013, Wang et al (45) established a nomogram using preoperative carcinoembryonic antigen levels and carbohydrate antigen19-9 levels, tumor size, presence or absence of vascular invasion, nodal status and direct invasion or local metastasis in a cohort of 367 ICC Asian patients. Furthermore, in 2014, Hyder et al (46) built a nomogram from a cohort of 514 patients from 13 Western and Eastern centers, in which the patients' age, tumor size, number of lesions, nodal status, vascular invasion status and presence of absence of underlying cirrhotic liver parenchyma were included. These two studies may predict the prognosis of patients with ICC and Doussot et al (47) verified this in 2015. This present study focused chiefly on the inflammation indicators for the prognosis of ICC and nomograms were used to verify the results. However, internal validation using the calibration curves and c-index demonstrated that the nomogram established in the present study was comparable with previous studies.

The present study poses a number of limitations. Firstly, the results were based on a single center retrospective study, which may generate biases in the data analysis. Secondly, two systems were established: The nomograms and the systemic inflammation-based system. Whilst these may effectively predict the prognosis of patients with ICC, the present study was unable to establish which one was superior. Thirdly, owing to the limited number of patients, further indicators, including GPS were not measured; hence, a full assessment of inflammatory indicators for ICC was not able to be made.
In the present study, it was demonstrated that low LMR and high NLR are associated with poor prognosis and worse clinical outcomes for patients with ICC undergoing curative surgery. A combined inflammation-based score system based on LMR and NLR may effectively predict the outcomes and serve as a novel prognostic predictor for these patients.

\section{Acknowledgements}

Not applicable.

\section{Funding}

This study was supported by the Project of Development and Innovation Team of Ministry of Education (grant no. IRT_16R57).

\section{Availability of data and materials}

The datasets used and/or analyzed during the current study are available from the corresponding author on reasonable request.

\section{Authors' contributions}

YW, YL and LH conceived and designed the experiments. YW, FR and ZX acquired data and performed statistical analysis. FR, YL, YC and CS interpreted the data. YW, LH, XZ and CS wrote the manuscript. XZ and YL revised the manuscript. XZ was involved in the acquisition of data. All authors approved the final version of the manuscript.

\section{Ethics approval and consent to participate}

All procedures performed in studies involving human participants were in accordance with the ethical standards of the institutional and/or national research committee and with the 1964 Helsinki declaration and its later amendments or comparable ethical standards. The institutional ethics committee at the study center approved this study. All participants gave consent after being fully informed of the goal and characteristics of this research.

\section{Patient consent for publication}

Written informed consent was obtained from all participants prior to publication.

\section{Competing interests}

The authors declare that they have no competing interests.

\section{References}

1. Nautsch F, Ludwig JM, Xing M, Johnson KM and Kim HS: Racial disparities and sociodemographic differences in incidence and survival among pediatric patients in the United States with primary liver cancer: A surveillance, epidemiology, and end results (SEER) population study. J Clin Gastroenterol 52: 262-267, 2018 
2. Xu K, Watanabe-Galloway S, Rochling FA, Zhang J, Farazi PA, Peng H, Wang $\mathrm{H}$ and Luo J: Practice, knowledge, and barriers for screening of hepatocellular carcinoma among high-risk chinese patients. Ann Glob Health 83: 281-292, 2017

3. He CB and Lin XJ: Inflammation scores predict the survival of patients with hepatocellular carcinoma who were treated with transarterial chemoembolization and recombinant human type-5 adenovirus H101. PLoS One 12: e0174769, 2017.

4. Hsu CS and Kao JH: An update on non-alcoholic fatty liver disease and non-alcoholic steatohepatitis in Asia. Expert Rev Gastroenterol Hepatol 11: 759-772, 2017.

5. Erstad DJ and Tanabe KK: Hepatocellular carcinoma: Early-stage management challenges. J Hepatocell Carcinoma 4 $81-92,2017$

6. Lee SJ and Lim HY: Hepatocellular carcinoma treatment: A comparative review of emerging growth factor receptor antagonists. Expert Opin Emerg Drugs 22: 191-200, 2017.

7. Rabinel P, Dousse D, Muscari F and Suc B: Management of liver cancer. The Surgeon's point of view. Rep Pract Oncol Radiother 22: 176-180, 2017

8. Rai V, Abdo J, Alsuwaidan AN, Agrawal S, Sharma P and Agrawal DK: Cellular and molecular targets for the immunotherapy of hepatocellular carcinoma. Mol Cell Biochem 437: 13-36, 2018

9. Jeong S, Cheng Q, Huang L, Wang J, Sha M, Tong Y, Xia L, Han L, Xi Z, Zhang J, et al: Risk stratification system to predict recurrence of intrahepatic cholangiocarcinoma after hepatic resection. BMC Cancer 17: 464, 2017

10. Zhu Y, Cai F, Zhao J and Liu F: Prognostic risk factors associated with recurrence and metastasis after radical resection in patients with hepatolithiasis complicated by intrahepatic cholangiocarcinoma. Cell Biochem Biophys 73: 455-460, 2015

11. Ni Q, Shen W, Zhang M, Yang C, Cai W, Wu M and Yang J: Prognostic analysis of radical resection for intrahepatic cholangiocarcinoma: A retrospective cohort study. Oncotarget 8: 75627-75637, 2017

12. Maganty K, Levi D, Moon J, Bejarano PA, Arosemena L, Tzakis A and Martin P: Combined hepatocellular carcinoma and intrahepatic cholangiocarcinoma: Outcome after liver transplantation. Dig Dis Sci 55: 3597-3601, 2010.

13. Elshamy M, Presser N, Hammad AY, Firl DJ, Coppa C, Fung J and Aucejo FN: Liver transplantation in patients with incidental hepatocellular carcinoma/cholangiocarcinoma and intrahepatic cholangiocarcinoma: A single-center experience. Hepatobiliary Pancreat Dis Int 16: 264-270, 2017.

14. Chinchilla-Lopez P, Aguilar-Olivos NE, Garcia-Gomez J, Hernández-Alejandro KK, Chablé-Montero F, Motola-Kuba D Patel T and Méndez-Sánchez N: Prevalence, risk factors, and survival of patients with intrahepatic cholangiocarcinoma. Ann Hepatol 16: 565-568, 2017.

15. Hanahan D and Weinberg RA: Hallmarks of cancer: The next generation. Cell 144: 646-674, 2011

16. Tarocchi M, Polvani S, Marroncini G and Galli A: Molecular mechanism of hepatitis B virus-induced hepatocarcinogenesis World J Gastroenterol 20: 11630-11640, 2014.

17. Song W, Wang K, Zhong FP, Fan YW, Peng L and Zou SB Clinicopathological and prognostic significance of platelet-tolymphocyte ratio in patients with hepatocellular carcinoma. Oncotarget 7: 81830-81838, 2016.

18. Fu SJ, Ji F, Han M, Chen MG, Wang XP, Ju WQ, Zhao Q, Wu LW, Ren QQ, Guo ZY, et al: Prognostic value of combined preoperative fibrinogen and neutrophil-lymphocyte ratio in patients with hepatocellular carcinoma after liver transplantation. Oncotarget 8: 4301-4312, 2017.

19. Howell J, Pinato DJ, Ramaswami R, Arizumi T, Ferrari C, Gibbin A, Burlone ME, Guaschino G, Toniutto P, Black J, et al: Integration of the cancer-related inflammatory response as a stratifying biomarker of survival in hepatocellular carcinoma treated with sorafenib. Oncotarget 8: 36161-36170, 2017.

20. Jing CY, Fu YP, Shen HJ, Zheng SS, Lin JJ, Yi Y, Huang JL, $\mathrm{Xu} \mathrm{X}$, Zhang J, Zhou J, et al: Albumin to gamma-glutamyltransferase ratio as a prognostic indicator in intrahepatic cholangiocarcinoma after curative resection. Oncotarget 8: 13293-13303, 2017

21. Okuno M, Ebata T, Yokoyama Y, Igami T, Sugawara G, Mizuno T, Yamaguchi J and Nagino M: Evaluation of inflammation-based prognostic scores in patients undergoing hepatobiliary resection for perihilar cholangiocarcinoma. J Gastroenterol 51: 153-161, 2016.
22. Meng ZW, Pan W, Hong HJ, Chen JZ and Chen YL: Modified staging classification for intrahepatic cholangiocarcinoma based on the sixth and seventh editions of the AJCC/UICC TNM staging systems. Medicine (Baltimore) 96: e7891, 2017.

23. Li Y, Jia H, Yu W, Xu Y, Li X, Li Q and Cai S: Nomograms for predicting prognostic value of inflammatory biomarkers in colorectal cancer patients after radical resection. Int J Cancer 139: 220-231, 2016.

24. Shi S, Chen Q, Ye L, Yin D, Li X, Dai Z and He J: Prognostic value of systemic inflammation score in patients with hepatocellular carcinoma after hepatectomy. Oncotarget 8: 79366-79375, 2017.

25. Hu D, Lin Y, Liu F, Zeng L, Ouyang X, Wang K, Zheng X and Huang Q: Elevated preoperative platelet to lymphocyte ratio indicates poor survival in patients with resected high-grade serous ovarian carcinoma. Clin Lab 62: 1443-1449, 2016.

26. Shi L, Qin X, Wang H, Xia Y, Li Y, Chen X, Shang L, Tai YT, Feng X, Acharya P, et al: Elevated neutrophil-to-lymphocyte ratio and monocyte-to-lymphocyte ratio and decreased platelet-to-lymphocyte ratio are associated with poor prognosis in multiple myeloma. Oncotarget 8: 18792-18801, 2017.

27. McMillan DC: Systemic inflammation, nutritional status and survival in patients with cancer. Curr Opin Clin Nutr Metab Care 12: 223-226, 2009.

28. Chen TH, Zhang YC, Tan YT, An X, Xue C, Deng YF, Yang W, Yuan $\mathrm{X}$ and Shi YX: Tumor-infiltrating lymphocytes predict prognosis of breast cancer patients treated with anti-Her-2 therapy. Oncotarget 8: 5219-5232, 2017.

29. Ji H, Houghton AM, Mariani TJ, Perera S, Kim CB, Padera R, Tonon G, McNamara K, Marconcini LA, Hezel A, et al: K-ras activation generates an inflammatory response in lung tumors. Oncogene 25: 2105-2112, 2006.

30. Shalapour S and Karin M: Immunity, inflammation, and cancer: An eternal fight between good and evil. J Clin Invest 125: 3347-3355, 2015.

31. Maeda K, Shibutani M, Otani H, Nagahara H, Ikeya T, Iseki Y, Tanaka H, Muguruma K and Hirakawa K: Inflammation-based factors and prognosis in patients with colorectal cancer. World J Gastrointest Oncol 7: 111-117,2015.

32. Beltran BE, Aguilar C, Quiñones P, Morales D, Chavez JC, Sotomayor EM and Castillo JJ: The neutrophil-to-lymphocyte ratio is an independent prognostic factor in patients with peripheral T-cell lymphoma, unspecified. Leuk Lymphoma 57: 58-62, 2016

33. Wu Y, Li C, Zhao J, Yang L, Liu F, Zheng H, Wang Z and Xu Y: Neutrophil-to-lymphocyte and platelet-to-lymphocyte ratios predict chemotherapy outcomes and prognosis in patients with colorectal cancer and synchronous liver metastasis. World J Surg Oncol 14: 289, 2016.

34. Hayashi H, Takamura H, Ohbatake Y, Nakanuma S, Tajima H, Fushida S, Onishi I, Tani T, Shimizu K and Ohta T: Postoperative changes in neutrophil-to-lymphocyte ratio and platelet count: A simple prognostic predictor for adult-to-adult living donor liver transplantation. Asian J Surg 41: 341-348, 2018.

35. Liu X, He L, Han J, Wang L, Li M, Jiang Y, Wang X and Yang Z: Association of neutrophil-lymphocyte ratio and $\mathrm{T}$ lymphocytes with the pathogenesis and progression of $\mathrm{HBV}$-associated primary liver cancer. PLoS One 12: e0170605, 2017.

36. Li SH, Wang QX, Yang ZY, Jiang W, Li C, Sun P, Wei W, Shi M and Guo RP: Prognostic value of the neutrophil-to-lymphocyte ratio for hepatocellular carcinoma patients with portal/hepatic vein tumor thrombosis. World J Gastroenterol 23: 3122-3132, 2017.

37. Zhang J, Zhang HY, Li J, Shao XY and Zhang CX: The elevated NLR, PLR and PLT may predict the prognosis of patients with colorectal cancer: A systematic review and meta-analysis. Oncotarget 8: 68837-68846, 2017.

38. Song X, Zhu H, Pei Q, Tan F, Li C, Zhou Z, Zhou Y, Yu N, Li Y and Pei H: Significance of inflammation-based indices in the prognosis of patients with non-metastatic colorectal cancer. Oncotarget 8: 45178-45189, 2017

39. Taussig MD, Irene Koran ME, Mouli SK, Ahmad A, Geevarghese S, Baker JC, Lipnik AJ, Banovac F and Brown DB: Neutrophil to lymphocyte ratio predicts disease progression following intra-arterial therapy of hepatocellular carcinoma. HPB (Oxford) 19: 458-464, 2017.

40. Wu SJ, Lin YX, Ye H, Li FY, Xiong XZ and Cheng NS: Lymphocyte to monocyte ratio and prognostic nutritional index predict survival outcomes of hepatitis B virus-associated hepatocellular carcinoma patients after curative hepatectomy. J Surg Oncol 114: 202-210, 2016. 
41. Song W, Tian C, Wang K, Zhang RJ and Zou SB: The pretreatment lymphocyte to monocyte ratio predicts clinical outcome for patients with hepatocellular carcinoma: A meta-analysis. Sci Rep 7: 46601, 2017.

42. Zhang C, Wang $\mathrm{H}$, Ning $\mathrm{Z}$, Xu L, Zhuang L, Wang P and Meng Z: Prognostic nutritional index serves as a predictive marker of survival and associates with systemic inflammatory response in metastatic intrahepatic cholangiocarcinoma. Onco Targets Ther 9: 6417-6423, 2016.

43. Jiang BG, Ge RL, Sun LL, Zong M, Wei GT and Zhang YJ: Clinical parameters predicting survival duration after hepatectomy for intrahepatic cholangiocarcinoma. Can J Gastroenterol 25: 603-608, 2011.

44. Zhang C, Wang H, Ning Z, Xu L, Zhuang L, Wang P and Meng Z: Serum liver enzymes serve as prognostic factors in patients with intrahepatic cholangiocarcinoma. Onco Targets Ther 10: 1441-1449, 2017.
45. Wang Y, Li J, Xia Y, Gong R, Wang K, Yan Z, Wan X, Liu G, Wu D, Shi L, et al: Prognostic nomogram for intrahepatic cholangiocarcinoma after partial hepatectomy. J Clin Oncol 31: 1188-1195, 2013

46. Hyder O, Marques H, Pulitano C, Marsh JW, Alexandrescu S, Bauer TW, Gamblin TC, Sotiropoulos GC, Paul A, Barroso E, et al: A nomogram to predict long-term survival after resection for intrahepatic cholangiocarcinoma: An Eastern and Western experience. JAMA Surg. 149: 432-438, 2014.

47. Doussot A, Groot-Koerkamp B, Wiggers JK, Chou J, Gonen M, DeMatteo RP, Allen PJ, Kingham TP, D'Angelica MI and Jarnagin WR: Outcomes after resection of intrahepatic cholangiocarcinoma: External validation and comparison of prognostic models. J Am Coll Surg 221: 452-461, 2015.

This work is licensed under a Creative Commons

Attribution-NonCommercial-NoDerivatives 4.0 International (CC BY-NC-ND 4.0) License. 